\title{
Differential Classical Conditioning of the Gill-Withdrawal Reflex in Aplysia Recruits Both NMDA Receptor-Dependent Enhancement and NMDA Receptor-Dependent Depression of the Reflex
}

\author{
Shekib A. Jami, ${ }^{1}$ William G. Wright, ${ }^{1,2}$ and David L. Glanzman ${ }^{1,3}$ \\ ${ }^{1}$ Department of Physiological Science, University of California, Los Angeles (UCLA), Los Angeles, California 90095-1606, ${ }^{2 B i o l o g i c a l ~ S c i e n c e s, ~ C h a p m a n ~}$ \\ University, Orange, California 92866, and ${ }^{3}$ Department of Neurobiology and the Brain Research Institute, David Geffen School of Medicine at UCLA, Los \\ Angeles, California 90095-1761
}

\begin{abstract}
Differential classical conditioning of the gill-withdrawal response (GWR) in Aplysia can be elicited by training in which a conditioned stimulus (CS) delivered to one side of the siphon (the CS+) is paired with a noxious unconditioned stimulus (US; tail shock), while a second conditioned stimulus (the $\mathrm{CS}-$ ), delivered to a different siphon site, is unpaired with the US. NMDA receptor (NMDAR) activation has been shown previously to be critical for nondifferential classical conditioning in Aplysia. Here, we used a semi-intact preparation to test whether differential classical conditioning of the GWR also depends on activation of NMDARs. Differential training produced conditioned enhancement of the reflexive response to the $\mathrm{CS}+$ and a reduction in the response to the $\mathrm{CS}-$. Comparison of the results after differential training with those after training in which only the two CSs were presented (CS-alone experiments) indicated that the decrement in the response to CS - after differential training was not caused by habituation. Surprisingly, differential training in the NMDAR antagonist APV (DL-2-amino-5-phosphonovalerate) blocked not only the conditioned enhancement of the GWR, but also the conditioning-induced depression of the GWR. We suggest that differential conditioning involves an NMDAR-dependent, competitive interaction between the separate neural pathways activated by the CS + and $\mathrm{CS}-$.
\end{abstract}

Key words: Aplysia; LTP; APV; synaptic competition; synaptic homeostasis; conditioned inhibition

\section{Introduction}

The marine snail Aplysia californica exhibits classical conditioning of its gill- and siphon-withdrawal reflex (SWR) (Carew et al., 1981, 1983; Antonov et al., 2003). This form of invertebrate associative learning is mediated, in part, by NMDA receptor (NMDAR)-dependent long-term potentiation (LTP) (Lin and Glanzman, 1994; Murphy and Glanzman, 1997; Antonov et al., 2003; Roberts and Glanzman, 2003). Previous work on the role of NMDAR-dependent LTP in classical conditioning in Aplysia has used mostly nondifferential conditioning protocols (but see Murphy and Glanzman, 1999) in which separate groups of animals (or preparations) receive either associative or nonassociative training. In differential conditioning in Aplysia, in contrast,

Received June 20, 2006; revised Jan. 25, 2007; accepted Jan. 26, 2007.

This work was supported by National Institutes of Health Grant NS29563 (D.L.G.), and National Science Foundation Grant IBN-0131743 (W.G.W., S.A.J.). We thank Drs. Daniel Fulton, Franklin Krasne, and Adam Roberts for helpful comments on this manuscript.

Correspondence should be addressed to Dr. David L. Glanzman, Gonda (Goldschmied) Neuroscience and Genetics Research Center, University of California, Los Angeles, 695 Young Drive South, Box 951761, Los Angeles, CA 900951761. E-mail: dglanzman@physci.ucla.edu.

S. A. Jami's present address: Postbaccalaureate Program, University of California Davis School of Medicine, One Shields Avenue, Davis, CA 95616.

DOI:10.1523/JNEUROSCI.2581-06.2007

Copyright $\odot 2007$ Society for Neuroscience $\quad$ 0270-6474/07/273064-05\$15.00/0 each animal receives two different conditioned stimuli (CSs), one of which is paired and the other unpaired, with the unconditioned stimulus (US) (Carew et al., 1983). Therefore, during differential conditioning the animal learns to expect the occurrence of the US when the CS + is presented, and its absence when the CS - is presented. Here, we asked whether activation of NMDARs, known to be critical for nondifferential behavioral conditioning in Aplysia (Antonov et al., 2003), is also necessary for differential classical conditioning. We observed that differential conditioning both enhanced the siphon-elicited gillwithdrawal response (GWR) to the CS+ and depressed the GWR to the CS - . We ruled out habituation as an explanation for the conditioning-induced reduction in the GWR to the CS- in additional experiments in which two different CSs were presented without the US. Interestingly, differential training in the presence of the NMDAR antagonist DL-2-amino-5-phosphonovalerate (APV) blocked both the enhancement of the GWR to the CS+ and the depression in the response to the CS-.

Some of our results have been published previously in abstract form (Jami et al., 2003).

\section{Materials and Methods}

Animals. Adult Aplysia californica (100-150 g) were obtained from Alacrity Marine Biological (Redondo Beach, CA). Animals were housed in a 


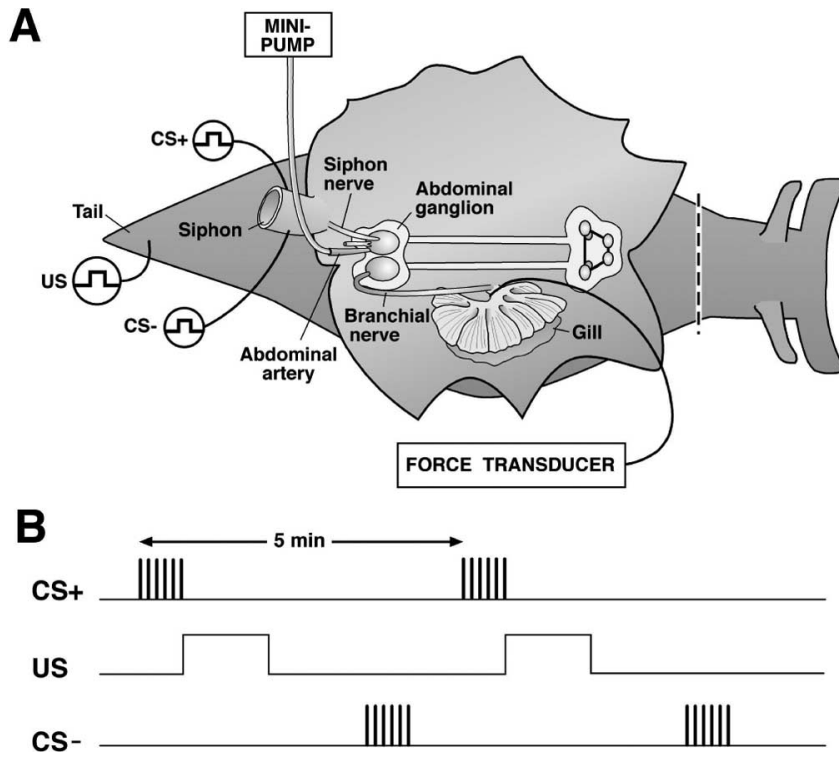

Figure 1. Preparation and experimental protocol. $A$, Illustration of the reduced preparation of Aplysia used for differential classical conditioning of the siphon-elicited GWR. $\boldsymbol{B}$, Illustration of the differential conditioning protocol. Training consisted of five total bouts of paired stimulation in which stimulation of one side of the siphon $(\mathrm{CS}+)$ was paired with the US. (The onset of the US occurred at the end of the CS.) The bouts of paired CS +-US stimulation occurred at one per $5 \mathrm{~min}$. Stimulation of the other side of the siphon (CS-) occurred $2.5 \mathrm{~min}$ after each occurrence of the paired stimulation. In other experiments, the preparations received training in which only the CSs were delivered (CS-alone experiments) (see Materials and Methods).

50 gallon aquarium filled with cooled $\left(14^{\circ} \mathrm{C}\right)$, aerated artificial seawater (ASW; Instant Ocean; Aquarium Systems, Mentor, OH). All animals were housed for $\geq 24 \mathrm{~h}$ before the start of the experiment.

Preparation. The semi-intact preparation was similar to that described previously (Ezzeddine and Glanzman, 2003) (Fig. 1). During experiments, the abdominal artery was perfused with aerated ASW $\left(15^{\circ}\right)$ via a cannula at a rate of $\sim 1 \mathrm{ml} / \mathrm{h}$. The cannula was used to selectively administer drugs to the abdominal ganglion. Stimulating electrodes (see below) were implanted into the siphon and tail. The afferent vein of the gill was cannulated and perfused with chilled, aerated ASW at a rate of $100 \mathrm{ml} / \mathrm{h}$. The cannula in the afferent vein was secured with a surgical silk suture and connected to a force transducer (UFI, Moro Bay, CA), which was used to measure the GWR. After setting up the preparation for stimulation and recording, it was rested for $\sim 1.5 \mathrm{~h}$. During experiments, the preparation was perfused with ASW $\left(13^{\circ}-15^{\circ}\right)$ at a rate of $1 \mathrm{~L} / \mathrm{h}$.

Stimulation. The conditioned and unconditioned stimuli were delivered via pairs of implanted Teflon-insulated platinum wires $(125 \mu \mathrm{m}$ in diameter; catalog \#773000; A-M Systems, Carlsborg, WA). Two pairs of wires were implanted in the siphon for delivery of the CSs, one pair on each side. A single pair of wires was implanted in the tail for delivery of the US. The stimuli used for the CS and US were $10 \mathrm{~ms}$ pulses of $25 \mathrm{~Hz}$ direct current (CS, $500 \mathrm{~ms}$ duration; US, $1 \mathrm{~s}$ duration) delivered by an electronic stimulator (S48; Grass-Telfactor, West Warwick, RI). The threshold voltage to elicit a GWR was determined for each of the three implanted electrodes (the two siphon electrodes and the tail electrode) $\sim 15$ min before the start of the experiment. (The threshold voltage for elicitation of the GWR in the experiments was 9-11 V. All preparations in which a stimulus of $>11 \mathrm{~V}$ was required to elicit the GWR were discarded.) The CS voltage was then set to be slightly above threshold level. The US was set at $\sim 5$ times the threshold level (range, $20-50 \mathrm{~V}$ ). The intensity of the US was fixed for a given experiment.

Experimental design. Three pretest stimuli were given to each siphon site at $2.5 \mathrm{~min}$ intervals. Some preparations then received differential classical conditioning training, beginning $2.5 \mathrm{~min}$ after the last pretest. Differential training consisted of five bouts of paired stimulation, in which stimulation of one of the siphon sites (the CS+) was paired with the US. The onset of the US was timed to occur at the offset of the CS
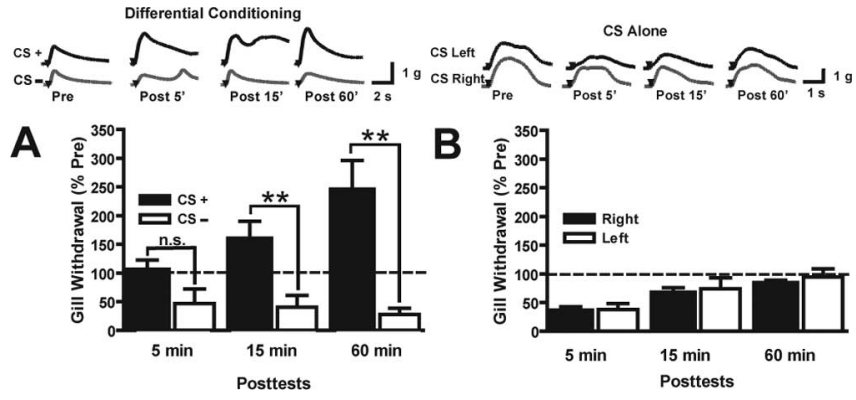

Figure 2. Differential classical conditioning of the GWR. A, Results for differential training in normal ASW. The graph shows the post-test results normalized to the results on the first pretest. Asterisks indicate statistical significance of the difference between the $C S+$ and $C S$ - results. Traces shown above the graph in this and Figure 3 represent GWRs recorded with the force transducer during one representative experiment. $\boldsymbol{B}$, Results for the CS-alone experiments.

(Carew et al., 1981, 1983). The side of the siphon, whether right or left, that received the CS+ stimulation was determined in pseudorandom manner before training. Bouts of paired CS+-US stimulation were applied at once per $5 \mathrm{~min}$. Stimulation of the second siphon site (the CS-) occurred $2.5 \mathrm{~min}$ after each bout of CS+-US stimulation (Fig. 1). The $\mathrm{CS}-$ stimulation was delivered five times. Thus, during differential conditioning, each preparation received each of the three stimuli (the CS+, the CS-, and the US) five times. Other preparations received training with only the CS (CS-alone training) after the pretest stimulation. Here, each siphon site received CS stimulation five times; the stimuli were alternately delivered to each siphon site (right or left) at 2.5 min intervals.

After the end of training, each siphon site received three post-test stimuli; the post-tests occurred at 5, 15, and $60 \mathrm{~min}$ after the final bout of CS+-US stimulation in the conditioning experiments, or after the last $\mathrm{CS}$ in the CS-alone experiments.

Drug application. In some preparations, training was performed in the presence of the NMDAR antagonist APV (Sigma, St. Louis, MO). The drug was perfused into the abdominal ganglion (Fig. 1). Perfusion with the drug began $15 \mathrm{~min}$ before the first pretest. Control preparations, performed at the same time as the experiments with APV, underwent training with normal ASW perfused into the abdominal ganglion. Preparations were assigned to training in either the drug or ASW according to a pseudorandom selection procedure.

Statistics. The GWR was normalized to the first pretest. The normalized data are expressed as means \pm SEM. A commercial software program (GraphPad Prism; GraphPad Software, San Diego, CA) was used for the statistical comparisons. Paired, within-group comparisons were performed with $t$ tests. Unpaired comparisons were performed with nonparametric Mann-Whitney tests because of significant differences among the group variances. All reported significance values represent two-tailed levels of significance.

\section{Results}

Differential conditioning produces enhancement of the GWR to the CS+ and decrement of the GWR to the CS-

Weak electrical stimulation of one side of the siphon (CS+) was paired with strong electrical shocks to the tail (the US); weak stimulation of the other side of the siphon (CS-) was unpaired with the US. The differential training resulted in a significant increase in the response to the CS+ compared with the response to the CS- (Fig. $2 A$ ) on the 15 and 60 min post-tests, but not on the 5 min test $(n=7)$. The mean normalized response to the CS+ was $102.6 \pm 20.0 \%$ on the 5 min post-test, $156.7 \pm 33.3 \%$ on the $15 \mathrm{~min}$ post-test, and $242.3 \pm 54.0$ on the $60 \mathrm{~min}$ post-test. The mean normalized response to the CS - was $46.8 \pm 25.4 \%$ on the 5 min post-test $(p>0.1), 40.3 \pm 20.5 \%$ on the 15 min post-test $(p<0.05)$, and $27.6 \pm 10.7 \%$ on the 60 min post-test $(p<$ $0.009)$. Paired $t$ tests indicated that the magnitude of the CS+ GWR was significantly enhanced on the 60 min post-test $(p<$ 
0.05) compared with its value on the initial pretest (pretest 1). Furthermore, the CS - GWR was significantly reduced compared with its pretest 1 value on the 15 and 60 min post-tests $(p<0.05$ for each comparison).

Weak stimulation of the siphon produces habituation of the GWR (Pinsker et al., 1970; Ezzeddine and Glanzman, 2003). We observed significant habituation of the GWR in additional experiments in which the same number of CSs were alternately given to the siphon at the same rate of stimulation used for differential conditioning, but in which the US was withheld (CS-alone experiments, $n=10$ ). Before comparing the pretest and post-test responses, we first statistically compared the GWRs to stimulation of the right and left sides of the siphon. There were no significant differences on any of the tests; we therefore combined the pretest and post-test data for the GWR to the right-side and left-side siphon stimulation for subsequent statistical comparisons. After CS-alone training, the mean GWR to the CS (combined rightside and left-side stimulation) was $35.1 \pm 7.1 \%$ on the $5 \mathrm{~min}$ post-test, $68.9 \pm 10.9 \%$ on the 15 min post-test, and $87.5 \pm 8.2 \%$ on the 60 min post-test (Fig. 2 B). Each of the post-test GWRs was also compared with the GWR on pretest 1 . The CS-alone stimulation produced significant habituation on the $5 \mathrm{~min}(p<0.001)$ and $15 \mathrm{~min}(p<0.02)$ post-tests, but not on the 60 min post-test $(p>0.1)$. Thus, by $1 \mathrm{~h}$ after the end of CS-alone stimulation, the GWR had returned to approximately its original strength, indicating that the habituation had worn off by this time.

To address the question of whether the depression of the response to the CS - during differential conditioning might be attributable to habituation, we statistically compared the differential conditioning results with those obtained using CS-alone stimulation. The CS-alone experiments were performed at the same time, and used animals from the same shipments as the differential conditioning experiments. To ensure validity of statistical comparisons between the post-test data from the two experimental groups, we compared the un-normalized pretest 1 GWRs in the two groups. The mean un-normalized GWR on pretest 1 was $2.8 \pm 0.5 \mathrm{~g}$ in preparations subsequently subjected to differential conditioning training, and $4.1 \pm 0.5 \mathrm{~g}$ in preparations that received only CS stimulation; these values were not significantly different ( $p>0.3$ ). Comparisons of the post-test results indicated that the $\mathrm{CS}+\mathrm{GWR}$ was significantly greater than the mean CS-alone GWR for all post-tests $(p<0.0005$ for the 5 min post-test, $p<0.003$ for the 15 min post-test, and $p<$ 0.001 for the $60 \mathrm{~min}$ post-test). Therefore, the paired training produced an increase in the GWR that persisted for $\geq 1 \mathrm{~h}$ after the end of training. The CS - GWR was significantly less than the mean CS-alone GWR for the 60 min post-test ( $p<0.003)$, although not for the earlier post-tests $(p>0.05)$. Consequently, the reduced GWR $1 \mathrm{~h}$ after the unpaired stimulation in the differential conditioning experiments cannot be attributed to habituation; rather, it represents a response decrement produced by the differential conditioning training.

\section{Differential conditioning of the GWR requires NMDAR activation}

A previous study showing involvement of NMDARs in classical conditioning in Aplysia used a nondifferential training protocol (Antonov et al., 2003). We therefore tested whether differential conditioning also involves NMDAR activation, as suggested by a previous electrophysiological study by Murphy and Glanzman (1999). Accordingly, we performed two more experiments: a control experiment in which preparations received differential training in normal ASW, and another experiment in which the
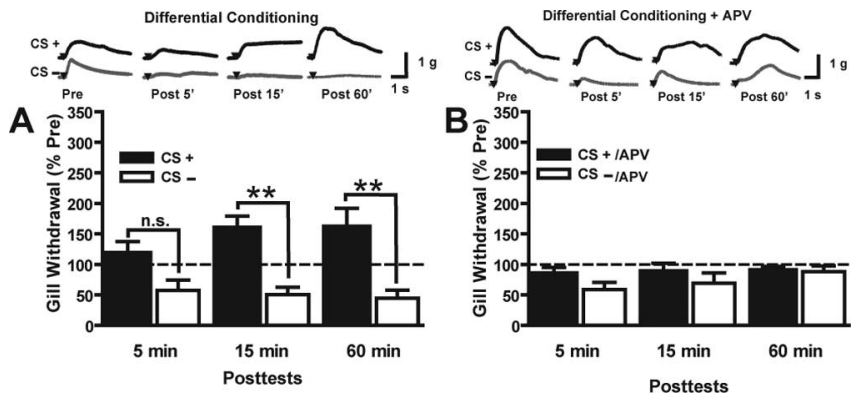

Figure 3. APV disrupts differential conditioning of the GWR. $A$, Results for the control experiment in which differential training was performed in ASW, similar to the experiment shown in Figure $2 B$. See the legend for Figure 2. Asterisks indicate statistical significance of the difference between the $C S+$ and $C S$ - results. $\boldsymbol{B}$, Results for experiments in which differential training was performed in APV (100 $\mu \mathrm{m})$; compare with the results in $\boldsymbol{A}$. n.S., Not significant.

preparations were differentially trained in the presence of APV (100 $\mu \mathrm{M}$; the drug was selectively infused into the abdominal ganglion). These two experiments were performed during the same period and used animals from the same shipments. As before, differential training produced a significant difference between the GWR evoked by the CS+ and that evoked by the CS$(n=10)$. The mean normalized GWR to the CS+ was $116.0 \pm$ $21.5 \%$ on the $5 \mathrm{~min}$ post-test, $157.3 \pm 22.1 \%$ on the $15 \mathrm{~min}$ post-test, and $159.2 \pm 32.6 \%$ on the 60 min post-test (Fig. $3 A$ ). In contrast, the normalized GWR to the CS - was $57.4 \pm 16.9 \%$ on the $5 \mathrm{~min}$ post-test $(p>0.05), 50.7 \pm 12.0 \%$ on the $15 \mathrm{~min}$ post-test $(p<0.01)$, and $44.9 \pm 13.0 \%$ on the 60 min post-test $(p<0.01)$. In addition, the paired stimulation produced a significant increase in the CS+GWR, compared with its pretest value, on the 15 min post-test ( $p<0.05$ ), although not on the 5 and 60 min post-tests. After unpaired stimulation, the CS - GWR was significantly reduced on all three post-tests compared with its pretest value $(p<0.05)$.

Training in APV blocked the differential conditioning of the GWR $(n=9)$. The mean normalized GWR to the CS+ was $82.5 \pm 13.0 \%$ on the $5 \mathrm{~min}$ post-test, $86.0 \pm 15.8 \%$ on the $15 \mathrm{~min}$ post-test, and $87.6 \pm 9.2 \%$ on the $60 \mathrm{~min}$ post-test (Fig. $3 B$ ). The CS+/APV GWR was not significantly different from its pretest value $(100 \%)$ for any of the post-tests $(p>0.05$ for each comparison). Also, the CS+/APV GWR was not significantly different from the CS-/APV GWR on the post-tests. The CS-/APV GWR was $58.6 \pm 11.8 \%$ on the 5 min post-test, $69.3 \pm 17.0 \%$ on the $15 \mathrm{~min}$ post-test, and $87.8 \pm 9.7 \%$ on the $60 \mathrm{~min}$ post-test ( $p \geq 0.1$ for the comparison with the response to the CS+/APV on each post-test). The CS-/APV GWR was significantly smaller on the 5 min post-test $(p<0.05)$ than on pretest 1 , but not on the later post-tests. Therefore, differential training in the presence of the NMDAR antagonist blocked not only conditioned enhancement of the GWR to the CS+, but also the reduction of the GWR to the CS-observed $\geq 15$ min after differential training in normal ASW.

The CS+ GWR after differential training in ASW was significantly greater than that after training in APV for the $15 \mathrm{~min}$ and 60 min post-tests ( $p<0.03$ and $p<0.02$, respectively), although not for the $5 \mathrm{~min}$ post-test $(p>0.2)$. Furthermore, the CSGWR after differential training in ASW was significantly less than the CS- GWR after differential training in APV on the $60 \mathrm{~min}$ post-test $(p<0.02)$. [The CS - GWR did not differ significantly between the groups after differential training in ASW and APV on the earlier post-tests $(p \geq 0.4)$.] Notice that the GWR to both the CS+ and CS - after differential training in APV resembled 
the GWR after CS alone stimulation on the 15 and 60 min posttests (Figs. $2 B, 3 B$ ). This result reinforces the idea that activation of NMDARs is critical for both the enhancement and reduction of the GWR observed after differential training in normal ASW.

\section{Discussion}

The present experiments demonstrate that differential conditioning of the GWR triggers both facilitatory and inhibitory processes. Reflexive withdrawal to the CS that was paired with the US (the CS + ) increased beyond the pretraining baseline, whereas, in the same preparations, withdrawal to the CS that was unpaired with the US (the CS-) decreased below the baseline. These differences could not be attributed to habituation: the GWR was more enhanced at 15 and $60 \mathrm{~min}$ after CS + training than it was at the same time points after CS alone training; moreover, the GWR was more diminished at $60 \mathrm{~min}$ after $\mathrm{CS}-$ training than it was 60 min after CS alone training. Both the facilitatory and inhibitory consequences of differential conditioning were abolished by the NMDAR antagonist APV. A variety of studies have indicated that associative learning can code for positive as well as negative correlations of stimuli across time (Wagner and Rescorla, 1972). In our study, a positive stimulus correlation, a CS + paired with the US, increased the strength of the reflex, whereas a negative correlation, a CS - specifically unpaired with the US, diminished its strength. The disruption of both of these effects by APV suggests a common mechanistic link between them. One mechanism that could account for our results is a homeostatic process in which the strength of multiple synaptic inputs onto a single target is regulated, such that overall synaptic strength is conserved. Evidence for the operation of such a homeostatic process has come mostly from studies of cell culture systems or development (Glanzman et al., 1991; Schacher et al., 1997; Turrigiano and Nelson, 2004); there is a paucity of evidence for homeostatic regulation of synaptic strength in studies of learning-related systems. Interpreted as the effect of homeostatic regulation, the decrement we observed in the anticorrelated CS - pathway reflects a competitive loss of synaptic strength because of the increase in strength in the coactive CS+ pathway.

An alternative explanation of our data is that the depression observed in the CS - pathway is caused by heterosynaptic inhibition. According to this nonhomeostatic model, an inhibitory interneuronal pathway activated by the US depresses all synaptic inputs except those that happen to be active during the US. This scheme posits an associative mechanism that enhances inputs coactive with the US, and a nonassociative mechanism that inhibits all, or most, of the other inputs.

Which of these mechanistic models is likely to be correct? The amplitude of the GWR on the final (60 min) trial after CS alone training fell between the amplitudes of the CS+ GWR and CSGWR on the same trial after differential conditioning (Fig. $2 A, B)$. Therefore, application of the US is required for the changes in both the CS + and CS - pathways. But both the homeostatic competition and the heterosynaptic inhibition models can account for these results. A control experiment that would serve to distinguish between the two models is training with unpaired CS and US stimulation (CS - training), but without the $\mathrm{CS}+$ stimulation (i.e., nondifferential $\mathrm{CS}-$ training). If the mechanism underlying the present data is synaptic competition caused by homeostatic regulation, then competitive inhibition of the CS - pathway should be reduced or abolished in nondifferential CS - training. This is because such training does not included a CS+, or, more correctly, an explicit CS + , and therefore the synaptic competition from coactive CS + inputs should be absent, or at least significantly reduced.

Such nondifferential CS- experiments have been performed previously in Aplysia. Murphy and Glanzman $(1997,1999)$ performed a cellular analog of classical conditioning of the withdrawal reflex. Brief activation of sensory neurons was either paired with tail nerve shock or specifically unpaired with nerve shock. Both differential conditioning analogs (Murphy and Glanzman, 1999) and nondifferential conditioning analogs (Murphy and Glanzman, 1997) were used, which enables a comparison between the effects of the two types of training. In their differential conditioning experiments, Murphy and Glanzman (1999) observed that the synaptic strength of the CS - pathway was depressed relative to its pretraining strength. Furthermore, the negative synaptic consequence of CS - training in the paradigm was significantly greater than that of CS - training in the nondifferential paradigm [Murphy and Glanzman (1999), their Fig. 6]. Thus, the inclusion of a specifically coactive CS+ pathway in the training protocol served to depress the CS - pathway more than would be expected by the unpaired training alone (see also Hawkins et al., 1998). These results support the idea that synaptic competition per se causes a decrement in the strength of unpaired pathways.

Most behavioral analyses of classical conditioning in Aplysia have used the siphon or mantle-elicited SWR (Hawkins et al., 1983, 1986; Antonov et al., 2001, 2003). None of these previous experiments has detected an inhibitory effect in the CS - pathway. However, a previous study of classical conditioning of the GWR by Carew et al. (1981) found an inhibitory effect on the CS - pathway similar to that reported here. In particular, the CS - pathway showed significant depression at both post-tests ( 5 and $60 \mathrm{~min}$ after training) relative to the pretraining level [Carew et al. (1981), their Fig. 8]. Interestingly, this study used a nondifferential training protocol. That significant depression was nonetheless observed in the CS- pathway would appear to argue against the synaptic competition hypothesis. However, this apparently negative evidence can actually be accommodated fairly easily within the framework of synaptic competition. Although no CS + is explicitly activated during nondifferential CS - training, this does not mean that synaptic inputs onto the motor neuron are silent during delivery of the US. In fact, motor neurons are massively depolarized by a variety of synaptic inputs during tail shock, primarily those from interneurons in the abdominal (Frost et al., 1988) and pleural (Cleary et al., 1995) ganglia. These inputs represent potential competition with the CS- sensory pathway for postsynaptic contact with gill motor neurons.

The results from our experiments with the NMDAR antagonist APV provide additional support for the idea that the decline in the strength of the CS - pathway is attributable to the enhancement of the CS+ pathway. In their synaptic experiments, Murphy and Glanzman (1999) found that differential conditioning produced significant enhancement of the CS + sensorimotor synapse and significant depression of the CS - sensorimotor synapse (60 min post-test). Furthermore, Murphy and Glanzman (1999) observed that training in APV eliminated both the enhancement of the CS + synapse and the depression of the CSsynapse [Murphy and Glanzman (1999), their Figs. 3, 4]. Murphy and Glanzman's APV results, like ours (Fig. 3), fit comfortably within the framework of homeostatic synaptic competition. (For another particularly compelling example of homeostatic synaptic competition, see Royer and Pare, 2003.)

Nonetheless, the alternative hypothesis for our data, that inhibition in the CS- pathway is unrelated to synaptic competi- 
tion, is difficult to exclude. For example, consider an additional control experiment, one in which nondifferential CS - training was performed in APV. The competitive homeostasis hypothesis would predict that the depression of the GWR observed by Carew et al. (1981) in their nondifferential CS - group would be absent if training were done in APV. This is because the competition from synapses that were (covertly) coactive with the US during training, and therefore potentiated, would be eliminated. But such an outcome would not allow one to definitively eliminate the alternative hypothesis. This is because there is currently no information regarding whether inhibitory pathways activated by tail shock in Aplysia (Mackey et al., 1987; Marcus et al., 1988) are sensitive to disruption of NMDAR activity. If these inhibitory pathways depend on NMDAR activation, their removal by APV would be indistinguishable from the removal of inhibition caused by homeostatic synaptic competition. Until the pharmacology of inhibition is better understood in Aplysia, therefore, it will be difficult to distinguish homeostatic from nonhomeostatic mechanistic hypotheses.

We do not understand why most previous studies of classical conditioning in Aplysia have failed to observe inhibition in the CS - pathway. Interestingly, the only behavioral studies that have reported such inhibition, the present study and that of Carew et al. (1981), have used the GWR rather than the SWR. Possibly, because the GWR is controlled by many fewer motor neurons than is the SWR (Kupfermann et al., 1974; Frost and Kandel, 1995), synaptic competition between paired (whether covertly or overtly) and unpaired inputs is more likely in the gill-withdrawal circuit than in the siphon-withdrawal circuit, where such competition may be more dispersed because of the greater number of postsynaptic targets. Siphon motor neurons were used in the synaptic conditioning studies of Murphy and Glanzman (1997, 1999); we do not have an explanation for the contrast between the behavioral results and synaptic results for the SWR system regarding the evidence for competition.

In summary, the GWR of Aplysia is a potentially useful system for studying the synaptic basis of inhibitory as well as excitatory learning. Our study provides a powerful experimental approach for understanding how facilitatory and inhibitory processes interact during classical conditioning, as well as the potential role of homeostatic mechanisms.

\section{References}

Antonov I, Antonova I, Kandel ER, Hawkins RD (2001) The contribution of activity-dependent synaptic plasticity to classical conditioning in Aplysia. J Neurosci 21:6413-6422.

Antonov I, Antonova I, Kandel ER, Hawkins RD (2003) Activity-dependent presynaptic facilitation and Hebbian LTP are both required and interact during classical conditioning in Aplysia. Neuron 37:135-147.

Carew TJ, Walters ET, Kandel ER (1981) Classical conditioning in a simple withdrawal reflex in Aplysia californica. J Neurosci 1:1426-1437.

Carew TJ, Hawkins RD, Kandel ER (1983) Differential classical conditioning of a defensive withdrawal reflex in Aplysia californica. Science 219:397-400.
Cleary LJ, Byrne JH, Frost WN (1995) Role of interneurons in defensive withdrawal reflexes in Aplysia. Learn Mem 2:133-151.

Ezzeddine Y, Glanzman DL (2003) Prolonged habituation of the gillwithdrawal reflex in Aplysia depends on protein synthesis, protein phosphatase activity, and postsynaptic glutamate receptors. J Neurosci 23:9585-9594.

Frost WN, Kandel ER (1995) Structure of the network mediating siphonelicited siphon withdrawal in Aplysia. J Neurophysiol 73:2413-2427.

Frost WN, Clark GA, Kandel ER (1988) Parallel processing of short-term memory for sensitization in Aplysia. J Neurobiol 19:297-334.

Glanzman DL, Kandel ER, Schacher S (1991) Target-dependent morphological segregation of Aplysia sensory outgrowth in vitro. Neuron 7:903-913.

Hawkins RD, Abrams TW, Carew TJ, Kandel ER (1983) A cellular mechanism of classical conditioning in Aplysia: activity-dependent amplification of presynaptic facilitation. Science 219:400-405.

Hawkins RD, Carew TJ, Kandel ER (1986) Effects of interstimulus interval and contingency on classical conditioning of the Aplysia siphon withdrawal reflex. J Neurosci 6:1695-1701.

Hawkins RD, Murphy GG, Glanzman DL (1998) Learning and the sensorimotor synapse in Aplysia. Science 281:619.

Jami SA, Wright WG, Glanzman DL (2003) Differential classical conditioning of the Aplysia gill-withdrawal reflex depends upon NMDA receptor activation and a competitive interaction between paired and unpaired pathways. Soc Neurosci Abstr 29:520.524.

Kupfermann I, Carew TJ, Kandel ER (1974) Local, reflex, and central commands controlling gill and siphon movements in Aplysia. J Neurophysiol 37:996-1019.

Lin XY, Glanzman DL (1994) Hebbian induction of long-term potentiation of Aplysia sensorimotor synapses: partial requirement for activation of an NMDA-related receptor. Proc Biol Sci 255:215-221.

Mackey SL, Glanzman DL, Small SA, Dyke AM, Kandel ER, Hawkins RD (1987) Tail shock produces inhibition as well as sensitization of the siphon-withdrawal reflex of Aplysia: possible behavioral role for presynaptic inhibition mediated by the peptide Phe-Met-Arg-Phe-NH2. Proc Natl Acad Sci USA 84:8730-8734.

Marcus EA, Nolen TG, Rankin CH, Carew TJ (1988) Behavioral dissociation of dishabituation, sensitization, and inhibition in Aplysia. Science 241:210-213.

Murphy GG, Glanzman DL (1997) Mediation of classical conditioning in Aplysia californica by LTP of sensorimotor synapses. Science 278:467-471.

Murphy GG, Glanzman DL (1999) Cellular analog of differential classical conditioning in Aplysia: disruption by the NMDA receptor-antagonist DL-2-amino-5-phosphonovalerate. J Neurosci 19:10595-10602.

Pinsker H, Kupfermann I, Castellucci V, Kandel E (1970) Habituation and dishabituation of the gill-withdrawal reflex in Aplysia. Science 167:1740-1742.

Roberts AC, Glanzman DL (2003) Learning in Aplysia: looking at synaptic plasticity from both sides. Trends Neurosci 26:662-670.

Royer S, Pare D (2003) Conservation of total synaptic weight through balanced synaptic depression and potentiation. Nature 422:518-522.

Schacher S, Wu F, Sun ZY (1997) Pathway-specific synaptic plasticity: activity-dependent enhancement and suppression of long-term heterosynaptic facilitation at converging inputs on a single target. J Neurosci 17:597-606.

Turrigiano GG, Nelson SB (2004) Homeostatic plasticity in the developing nervous system. Nat Rev Neurosci 5:97-107.

Wagner AR, Rescorla RA (1972) Inhibition in Pavlovian conditioning: application of a theory. In: Inhibition and learning (Boakes RA, Halliday MS, eds), pp 301-316. New York: Academic. 